\title{
The effect of a crack-tip radius on the validity of the singular solution
}

\author{
D Dini and D A Hills* \\ Department of Engineering Science, University of Oxford, Oxford, UK
}

\begin{abstract}
The influence of the finite crack-root radius on the local stress field at the root of a crack is found explicitly. This is then applied as an inner asymptotic solution, embedded with the conventional crack-tip singular solution, to quantify the possible influence of local rounding on the ability of the singular solution to capture the characteristics of the crack-tip process zone. The scaling factor employed is the conventional crack-tip stress intensity, and the example of a simple edge crack in a tension field is used to illustrate the method.
\end{abstract}

Keywords: crack-tip radius, singular solution, local stress field, stress intensity

\section{INTRODUCTION}

Linear elastic fracture mechanics hinges on the encapsulation of the crack-tip stress field by the stress intensity factor. The connection between the stress intensity factor and the strain energy release rate through the Irwin-Kolosov relation [1] demonstrates that Griffith's criterion is consistent with the stress intensity reaching a critical value. Of almost greater importance is the empirical correlation between the range of stress intensity experienced and the fatigue crack growth rate [2]. Although the correlation itself is empirically established, the underlying connection between the two hinges on the 'process zone', i.e. the zone of plasticity at the crack tip, where irreversibilities lead to the exhaustion of ductility and hence to the extension of the crack, being characterized by the elastic singular field. Thus, the key quantities influencing crack extension must be controlled by a substantial elastic hinterland where the stress state is given by the singular solution [3]. This is the basis for the proviso that 'smallscale yielding' should obtain in the crack-tip plastic region. A paradox of crack-tip behaviour is that the local implied singularity means that there must always be some plasticity; and this plasticity is responsible both for crack growth (under repeated loading) and resistance to crack growth. Crack-tip plasticity means that residual stresses are always present after the first cycle of load, at the crack tip, and these will both materially

\footnotetext{
The MS was received on 8 October 2003 and was accepted after revision for publication on 27 February 2004.

* Corresponding author: Department of Engineering Science, University of Oxford, Parks Road, Oxford OX1 3PJ, UK.
}

influence the local stress state and cause the crack faces either to remain apart (in the case of a stationary crack) or be pressed together (in the case of a propagating crack). Separately, the plastic deformation means that even an initially atomically sharp crack tip will become radiused.

There have been many studies, at increasing levels of sophistication, over recent years of the crack-tip plasticity problem [4], and most have focused on the form of the plastic zone and the nature of the residual stresses induced. This paper will not consider these aspects but address, instead, solely the question of the influence of crack-tip rounding on the nature of the local stress state. In particular, the question arises as to how big the radius at the end of a crack could be without invalidating the domination of the fatigue process by the singular stress field. The technique to be developed does not enquire how the crack-root end radius arose, and so is equally valid in assessing the effects of rounding present at the root of slots or 'starter notches', and is particularly suited to judging whether EDM-formed slots are cracklike in their characteristics, as notch roots formed this way are invariably almost semicircular in form.

The approach to be developed hinges on the use of a set of nested asymptotic elastic solutions, and a universal solution for permitted root radius, but ensuring singular response, will emerge. An example problem will be considered, as shown in Fig. 1A. This concerns the edge-breaking crack present on the surface of a half-plane and subject to uniform remote tension. Suppose that, firstly, the limit of small-scale yielding is to be determined. This could be found by taking the semi-infinite crack solution (that corresponding to the 


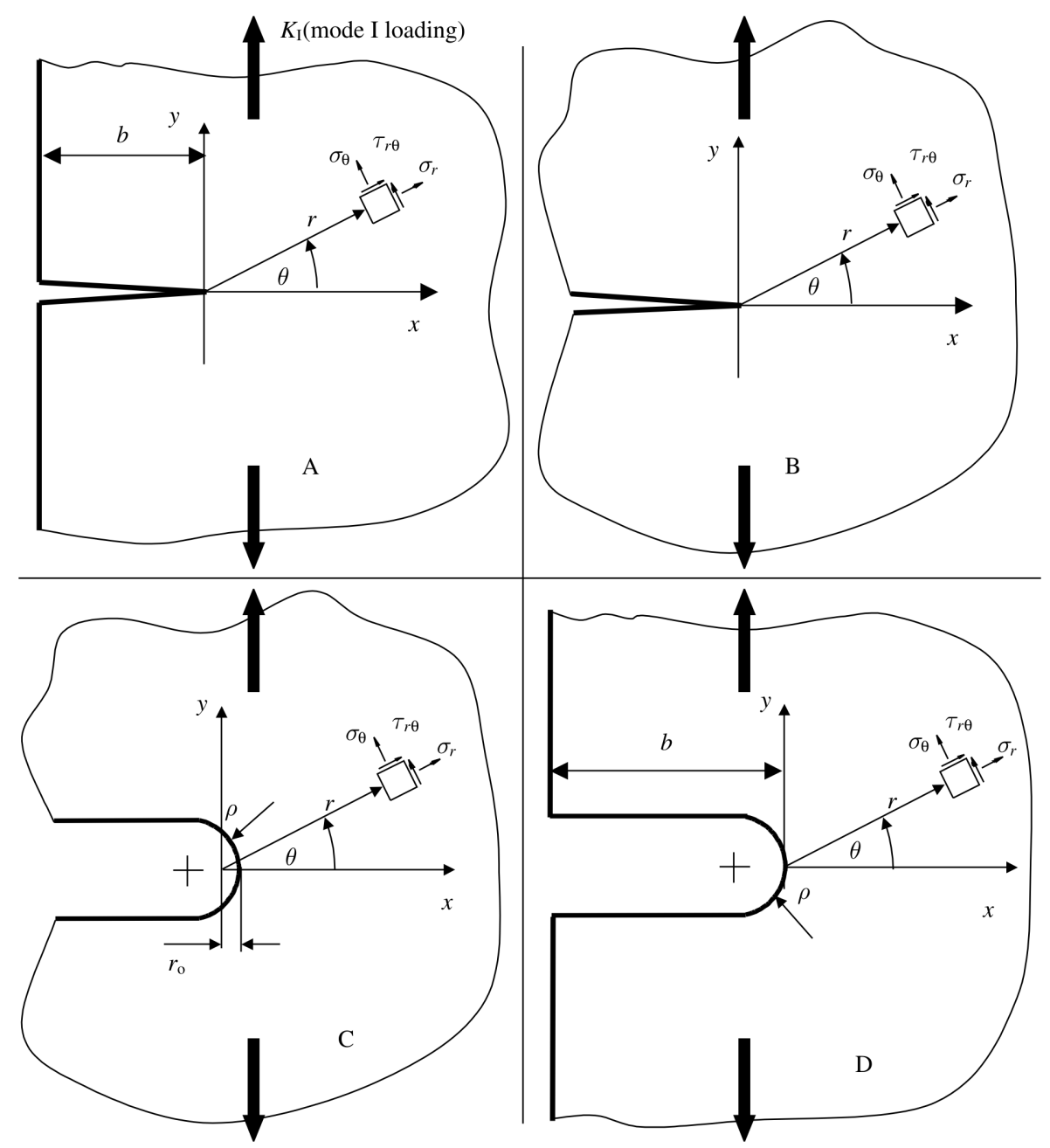

Fig. 1 (A) Edge crack in the example finite body, (B) semi-infinite edge crack, (C) rounded edge crack in the example finite body and (D) semi-infinite rounded edge crack

crack-tip stress intensity) (Fig. 1B), and noting the domain in which agreement with the finite crack solution is obtained to within a given degree of accuracy [5]. This would then limit the extent of the plastic zone that could be accommodated as a necessary condition is for the elastic hinterland to be characterized by the usual singular field, to within a given tolerance. At the same time, the stress intensity factor would emerge. The next phase of the procedure is to establish a solution for the semi-infinite rounded slot (Fig. 1C). It should be noted that, remote from the notch root, the stress field for this problem approaches that of a semi-infinite sharp crack (i.e. the solutions to problems B and C diverge when $r / \rho$ becomes small, adjacent to the notch root). The argument is made that, if the majority of the process zone experiences a strain field dominated by the elastic strains generated by an exterior singular field, the presence of deviation from this field near the inner boundary will have a small effect. The present authors argue that the exhaustion of ductility within the process zone will, under these conditions, still be controlled by the stress intensity factor. Note that this is despite the fact that at the notch root the strain state must be bounded while at the crack tip it must be singular. Thus, by noting the discrepancy between these two solutions, the lower extent of the process zone, given that it must be controlled by the singular field, may be found. This part of the method gives rise to a universal solution, which may be applied to any crack, without further calculation, once the stress intensity factor is known. Thus, the solution for a crack in a finite body but with a root radius (here Fig. 1D) may be inferred without having to solve the problem itself, within certain limitations.

\section{FORMULATION}

The enabling solutions, here, are those relating to the auxiliary problems (Figs 1B and C), and these are now given. 


\section{SEMI-INFINITE CRACK}

The solution for a semi-infinite crack suffering mode I loading is well known, and may be derived directly from Williams' solution for a semi-infinite wedge [6], and choosing the internal wedge angle to be $2 \pi \mathrm{rad}$, or by using a series expansion on, for example, the Westergaard [7] solution for a finite crack. Either way, the stress state may be written in the form

$$
\sigma_{i j}[r, \theta]=\frac{K_{\mathrm{I}}}{\sqrt{2 \pi r}} f_{i j}(\theta)
$$

where the spatial distribution is given by

$$
\left\{\begin{array}{l}
f_{\theta \theta} \\
f_{r r} \\
f_{r \theta}
\end{array}\right\}=\left[\frac{1}{2}\left[\left\{\begin{array}{c}
\frac{3}{2} \cos \left[\frac{\theta}{2}\right] \\
\frac{5}{2} \cos \left[\frac{\theta}{2}\right] \\
\frac{1}{2} \sin \left[\frac{\theta}{2}\right]
\end{array}\right\}+\frac{1}{2}\left\{\begin{array}{c}
\cos \left[\frac{3}{2} \theta\right] \\
-\cos \left[\frac{3}{2} \theta\right] \\
\sin \left[\frac{3}{2} \theta\right]
\end{array}\right\}\right]\right]
$$

and $K_{\mathrm{I}}$ is the stress intensity factor.

\subsection{Semi-infinite rounded slot}

This problem is depicted in Fig. 1C and is a special case of the semi-infinite radiused-root $\mathrm{V}$-notch recently analysed very effectively by Fillippi et al. [8], whose work should be consulted for details. The present paper merely records the relevant results for the stress field, for the special case where the notch sides are parallel, forming a 'slot', i.e. something geometrically equivalent to a crack, but with a tip radius. In polar coordinates, centred as shown in the figure [with $r_{0}=\rho(q-1) / q$ and $q=(2 \pi-2 \alpha) / \pi]$, the stress state is given by

$$
\sigma_{i j}(r, \theta)=\frac{K_{\mathrm{I}}}{\sqrt{2 \pi r}}\left[f_{i j}(\theta)+g_{i j}(r, \theta)\right]
$$

where the functions $g_{i j}(r, \theta)$, which may be thought of as the perturbation of the singular field, are given by

$$
\left\{\begin{array}{l}
g_{\theta \theta} \\
g_{r r} \\
g_{r \theta}
\end{array}\right\}=\left[\frac{\rho}{2 r}\left(\left\{\begin{array}{c}
\cos \left[\frac{\theta}{2}\right] \\
-\cos \left[\frac{\theta}{2}\right] \\
\sin \left[\frac{\theta}{2}\right]
\end{array}\right\}\right)\right]
$$

and $\rho$ is the crack tip radius.

\section{NESTING OF INNER AND OUTER ASYMPTOTES}

The two asymptotic solutions described above (problems B and C) have been set out in a way that makes it clear that, for large values of $r / \rho$, the two fields must tend to the same stress distribution, if $K_{\mathrm{I}}$ is the same for each. Thus, once the value of the stress intensity factor has been found for the outer asymptotic (the standard semi-infinite crack solution), the same scaling factor may be used to determine the inner stress field when a radius is present. It is therefore possible to compare the two asymptotic fields for the general case. Figure 2 displays, at two magnifications, the state of stress ahead of the 'crack' tip, and the contours of two quantities are given. One (shown in solid lines) is the location of the plastic front, or the process zone size, as a function of the normalized applied load $\left(K_{\mathrm{I}} / k \sqrt{\rho}\right)$, where $k$ is the yield stress in pure shear (only the half-plane $\theta \geq 0$ is shown for economy of space; the lower half-plane is, of course, symmetrical). The von Mises yield criterion has been assumed, and transverse plane strain, with Poisson's ratio set to 0.3 . The second set of contours (broken lines) displays the fractional mismatch between the inner and outer asymptotic solutions as characterized by the second deviatoric stress invariant (von Mises parameter). As expected, the fractional difference increases as the 'crack' tip is approached, but it does so in a very non-uniform way (in a polar sense), because the eigenfunctions $f_{i j}(\theta)$, relating to the outer asymptote, and $g_{i j}(r, \theta)$, relating to the inner asymptote, are very different in form.

Consider the form of the discrepancy contours qualitatively (see Fig. 2a). They show that, as $\theta \rightarrow \pm \pi$, i.e. along the flanks of the crack, the stress fields are quite different, even though the discrepancy must tend to zero for large values of $r / \rho$. The region (approximately) $\pi / 6<\theta<3 \pi / 4$ shows a remarkably consistent correlation between the two solutions, with a maximum absolute dicrepancy of about 5 per cent. As $\theta \rightarrow 0$ (ahead of the crack tip), the discrepancy again increases in magnitude, although the maximum value attained is much less than along the crack flanks.

Clearly, because of the considerable extent of the mismatched region as $\theta \rightarrow \pm \pi$, it is impossible to find any error contour circumscribed by a process zone front contour. It follows that it is impossible, no matter how small the 'crack-tip radius', for a regime to be found rigorously when the process zone strains are everywhere controlled by a singular elastic hinterland. It is proposed, therefore, to discount any attempt to match in this neighbourhood, but instead ensure that around the majority of the process zone front the strains are controlled by $K_{\mathrm{I}}$ (in practice $0 \leqslant \theta \lesssim 3 \pi / 4$ as shown in Fig. 2a, where the shaded region highlights the domain outside the chosen process zone front in which the discrepancy exceeds 5 per cent). The $K_{\mathrm{I}}$ dominated hinterland encompasses both the region in which, for 


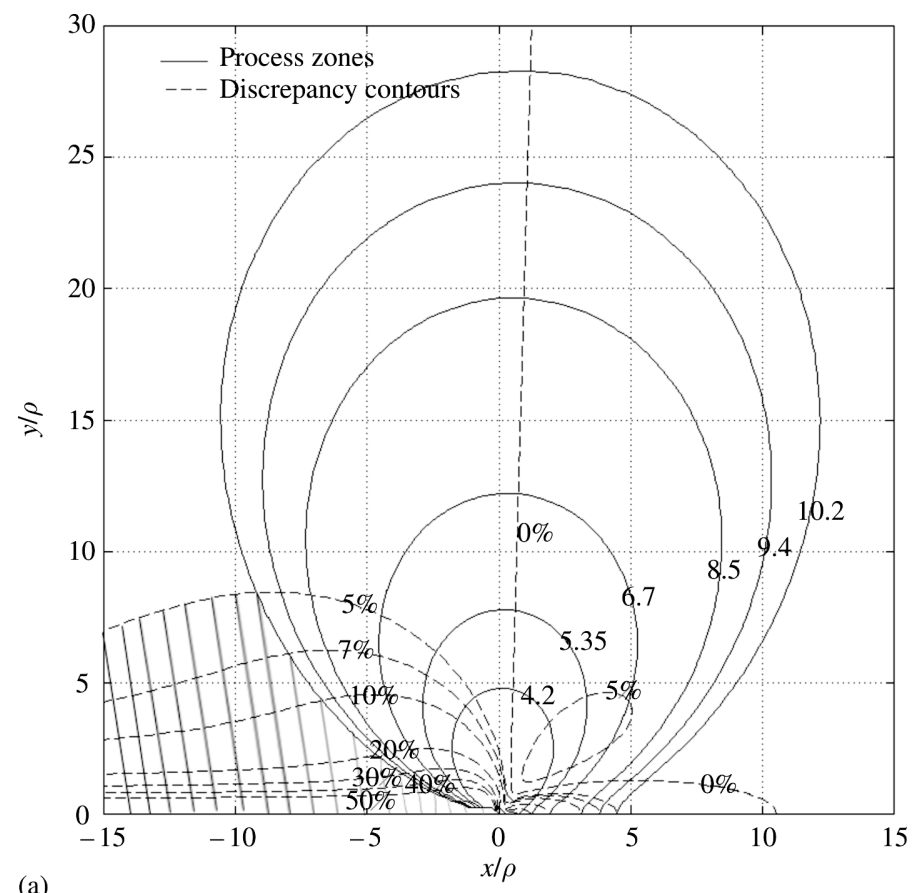

(a)

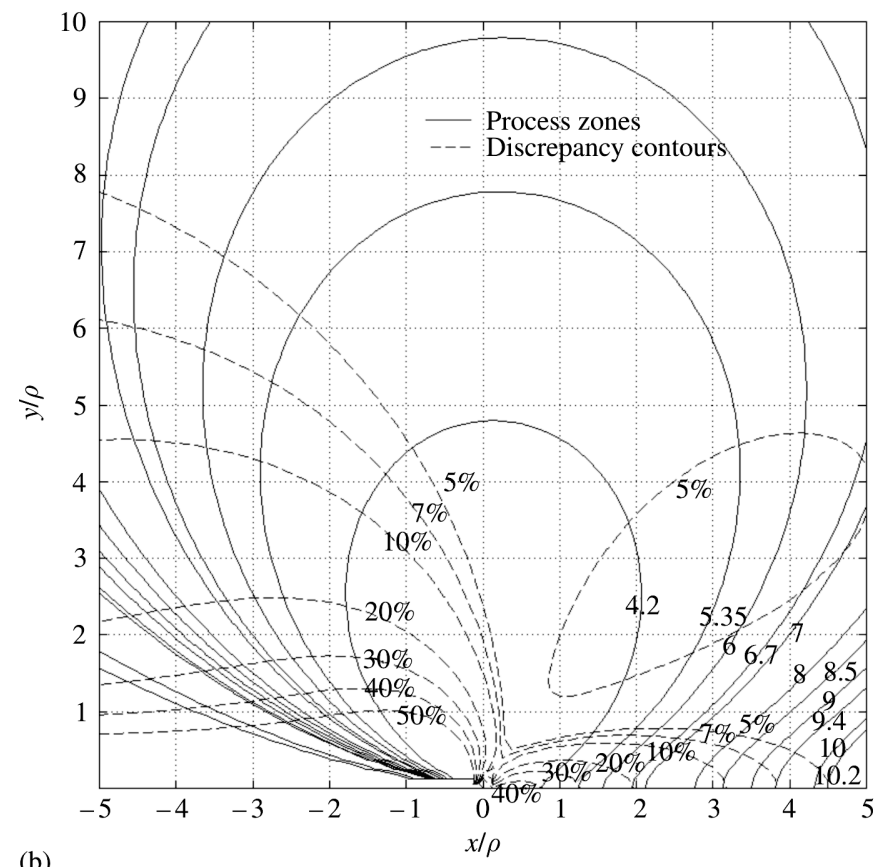

Fig. 2 Discrepancy between solutions for problems C and B as implied by the von Mises parameter, i.e.

$$
\left|\sqrt{J_{2}^{\mathrm{C}}}-\sqrt{J_{2}^{\mathrm{B}}}\right| / \sqrt{J_{2}^{\mathrm{C}}}
$$

together with process zone front locations, for a given normalized stress intensity factor, $K_{\mathrm{I}} / k \rho^{0.5}:$ (a) global view, (b) close-up

any given load, the process zone radius is largest and the region where the exhaustion of ductility leads to extension of the crack $(\theta \approx 0)$. In practice, the line $\theta=$ 0 therefore delineates the maximum mismatch incurred and the corresponding plastic front location (whose values are shown in Fig. 2) which may be controlled.
By comparing the location of the plastic front with the relative discrepancy in the stress state, as captured by the singular solution, it is accepted that the minimum load needed to achieve this degree of accuracy may be found. Note that this figure actually gives the mismatch implied by the elastic solutions at this point. In order to 
achieve this degree of absolute accuracy in the process zone, the elastic hinterland would have to extend beyond the plastic front by a considerable amount. Nevertheless, this procedure provides a good measure of the accuracy to be expected.

It is emphasized that, because each of the characteristic fields under consideration scales with the stress intensity factor, the comparison made in Fig. 2 is completely general in its application. In order to use it for a particular finite geometry, it is necessary only to find the value of $K_{\mathrm{I}}$ for a conventional sharp crack in that geometry.

\section{EXAMPLE PROBLEM: EDGE CRACK}

As an example in the use of the procedure described, consider the problem of an edge crack under remote uniform tension. This problem may be solved in a number of ways, but one that not only enables the crack tip stress intensity factor but also the complete local stress field to be found readily is the distributed dislocation method, which is described in detail by Nowell and Hills $[9,10]$ and only the briefest outline will be given here.

The first step of the procedure is to determine the state of stress, $\sigma_{i j}^{\mathrm{d}}$ (with $i, j=x, y$ ), induced by a single dislocation of magnitude $\boldsymbol{b}_{y}$ (the Burgers vector) placed at a point $\left(x_{\mathrm{d}}, 0\right)$ in a half-plane. Using results reported in reference [11], the stress components at a particular point $(x, y)$ are given by

$$
\sigma_{i j}^{\mathrm{d}}(x, y)=\frac{\mu}{\pi(k+1)} \boldsymbol{b}_{y}\left(x_{\mathrm{d}}\right) G_{y i j}\left(x, x_{\mathrm{d}}, y\right)
$$

where $\mu$ is the shear modulus and $k$ is Kolosov's constant [equal to $3-4 v$ in plane strain or $(3-v) /(1+v)$ in plane stress, where $v$ is Poisson's ratio] and the coordinate set for the half-plane is as shown in Fig. 1. The functions $G_{y i j}$ may be obtained from reference [9].

Rather than installing discrete dislocations, a continuous distribution of the Burgers vector density, $B_{y}$, is employed along the line of the crack, so that the stress due to this distribution, together with the farfield stress, $\sigma_{0}$, is given by

$$
\sigma_{i j}(x, y)=\sigma_{0}+\frac{\mu}{\pi(k+1)} \int_{0}^{b} B_{y} G_{y i j}\left(x, x_{\mathrm{d}}, y\right) \mathrm{d} x_{\mathrm{d}}
$$

Since the crack faces are traction free, the following Cauchy singular integral equation, applied along the line $y=0$, is obtained:

$$
\sigma_{0}+\frac{\mu}{\pi(k+1)} \int_{0}^{b} B_{y}\left(x_{\mathrm{d}}\right) G_{y y y}\left(x, x_{\mathrm{d}}, 0\right) \mathrm{d} x_{\mathrm{d}}=0
$$

where $G_{y y y}\left(x, x_{\mathrm{d}}, 0\right)$ can be rewritten in the simplified form

$$
\begin{aligned}
& G_{y y y}\left(x, x_{\mathrm{d}}, 0\right) \\
& \quad=2\left[\frac{1}{x-x_{\mathrm{d}}}-\frac{1}{x+x_{\mathrm{d}}}-\frac{2 x_{\mathrm{d}}}{\left(x+x_{\mathrm{d}}\right)^{2}}+\frac{4 x_{\mathrm{d}}^{2}}{\left(x+x_{\mathrm{d}}\right)^{3}}\right]
\end{aligned}
$$

This equation may not be inverted in closed form, and so a numerical procedure, using Gauss-Jacobi quadrature [12], is employed. This yields $n$ simultaneous linear algebraic equations, which may be solved using standard techniques. The procedure is as follows:

1. Normalize the range of integration to \pm 1 by an appropriate change in variables.

2. Consider the behaviour of the unknown function at the ends of the crack. For a crack of size $b$ whose origin is at the surface, such as that shown in Fig. 1A, the unknown function $B_{y}\left(x_{\mathrm{d}}\right)$ is singular at the crack tip, as $x \rightarrow b$, and bounded at the crack mouth, as $x \rightarrow 0$.

3. Represent the unknown function as a product of an appropriate weight function, $w$, which incorporates the expected behaviour at the end-points and a bounded function $\phi$. Since the singularity order is expected to be $r^{-0.5}$, the appropriate choice of weight function is

$$
w(u)=\sqrt{\frac{1+u}{1-u}}
$$

where $u$ is the normalized variable, bounded at $u=$ -1 and singular at $u=1$.

4. Gauss-Jacobi integration may now be used to reduce the integral in equation (7) to a set of simultaneous linear equations in order to obtain the values of the bounded function $\phi$ at $N$ integration points ( $N$ was set to 50 for good accuracy). The discretized form of equation (7) is

$$
\begin{aligned}
\sigma_{0}\left(v_{k}\right) & +\frac{\mu}{\pi(k+1)} \sum_{i=1}^{N} \frac{2\left(1+u_{i}\right)}{2 N+1} G_{y y y}\left(u_{i}, v_{k}, 0\right) \phi\left(u_{i}\right) \\
& =0
\end{aligned}
$$

where

$$
\begin{array}{ll}
u_{i}=\cos \left(\frac{2 i-1}{2 N+1} \pi\right), & i=1,2, \ldots, n \\
v_{k}=\cos \left(\frac{2 k}{2 N+1} \pi\right), & k=1,2, \ldots, n
\end{array}
$$

The solution of equation system (10) gives $n$ values of $\phi$ and hence $B_{y}(u)$, from which the crack tip stress intensity may be found by Krenk interpolation. Its value is that obtained by Kaya and Erdogan [13] and is

$$
\frac{K_{\mathrm{I}}}{\sigma_{0} \sqrt{\pi b}}=1.12152
$$


Once the values of the dislocation density are known, it is a routine matter to obtain the complete stress field by evaluation of equation (6) which now represents a simple integral. Note that, away from the crack itself, the integral is regular, and hence the choice of the field coordinates is free and is not constrained to those implied by the numerical quadrature scheme.

\section{SMALL-SCALE YIELDING}

The limit of small-scale yielding represents the largest load that may be applied while maintaining a good description of the crack-tip plastic zone by the stress intensity factor (in contrast to the minimum load calculated in section 4). Several measures of the quality of the singular field could be employed, but the one that has greatest relevance in the present context would seem to be the plasticity parameter itself. Also in contrast to the lower bound to the load, this result is, of course, geometry specific, and the results presented here are for the problem in Fig. 1A alone.

Accordingly, Fig. 3 gives plots incorporating two sets of contours. The first is the fractional mismatch between the von Mises parameter (the second invariant of the stress deviator), as measured by the full stress field and by the singular term only. The second set of contours shows the position of the plastic front for various values of applied load $\left(\sigma_{0} / k\right)$. Thus, for example, if a discrepancy in the characterization of the surrounding elastic crack tip stress field of \pm 5 per cent is to be permitted, the maximum load that may be applied, for this particular geometry, is $\sigma_{0} / k=0.036$, while, if a discrepancy of \pm 30 per cent is acceptable, the maximum load that may be applied goes up to $\sigma_{0} / k=$ 0.945 (see Fig. 3a).

\section{BOUNDS ON THE APPLIED LOAD}

This paper is concerned with the validity of the assumption that the crack tip process zone is characterized by the singular term, with and without local rounding. The maximum corresponds to the classic small-scale yielding requirement, and is, to repeat, a geometry dependent phenomenon. Using the results just found in the preceding section, this requirement may be summarized in the plot shown in Fig. 4, where the horizontal lines denote this limit. It is again emphasized that the term ' 5 per cent discrepancy' means that there is a mismatch in the elastic fields of this value, as characterized by the second deviatoric invariant, when their plastic front, as implied by the elastic solution, lies at this point. Thus, there is inaccuracy both in the estimated position of the plasticity front (which does not allow for stress redistribution) and because a significant region of elastic hinterland must also be controlled by the stress intensity solution to achieve the accuracy cited. Nevertheless, the procedure described provides a completely consistent procedure.

The second set of lines in the figure gives the lower bound to the permitted applied load and corresponds to the minimum load that can be tolerated when the singular sharp semi-infinite crack stress field and semiinfinite rounded crack tip field agree. These were derived by using the general results of matching displayed in Fig. 2 and applying the calibration for the stress intensity factor, $K_{\mathrm{I}}$, for this particular geometry [equation (13)]. The permitted load range for a given degree of accuracy decreases as the crack tip radius increases. For example, if a maximum discrepancy of 5 per cent is required, the load and dimensionless root radius must lie in the shaded region of Fig. 4. No solution to this degree of accuracy is possible if $\rho / b$ exceeds 0.000052 or $\sigma_{0} / k$ exceeds about 0.036 . If a mismatch of 7 per cent is acceptable, then $\rho / b$ must certainly be less than 0.000242 and $\sigma_{0} / k$ cannot exceed about 0.074 . These requirements are certainly stringent; with conversion to dimensional quantities, it suggests that, for the root radius to have less than a 7 per cent effect on the singular field, a $10 \mathrm{~mm}$ edge crack would need to have an end radius of less than $2.4 \mu \mathrm{m}$. Equally, the small-scale yielding assumption imposed at the same level of accuracy would imply a maximum remote load of 7 per cent of the yield stress in pure shear (see Fig. 4a). A less ambitious choice in terms of acceptable mismatch (e.g. 20 per cent) will instead lead to less stringent requirements in terms of both $\rho / b$ and $\sigma_{0} / k$, as shown in Fig. 4b.

\section{CONCLUSION}

The solution for a semi-infinite radiused-root crack has been found and used as an inner asymptote to embed within the conventional crack-tip singular solution. This enables the lower bound load for a given degree of accuracy in the characterization of the process zone to be found. These results are displayed in Fig. 2, and apply regardless of the geometry of the crack. As an example of their application, they have been used to reconcile the edge crack in tension. This underlines the stringent requirements on load range and crack-tip radius imposed by the small-scale yielding and lower bounds, and suggests that a wider tolerance of mismatch at the process zone front may be acceptable. Figure 5 therefore summarizes the requirements imposed in Fig. 2 


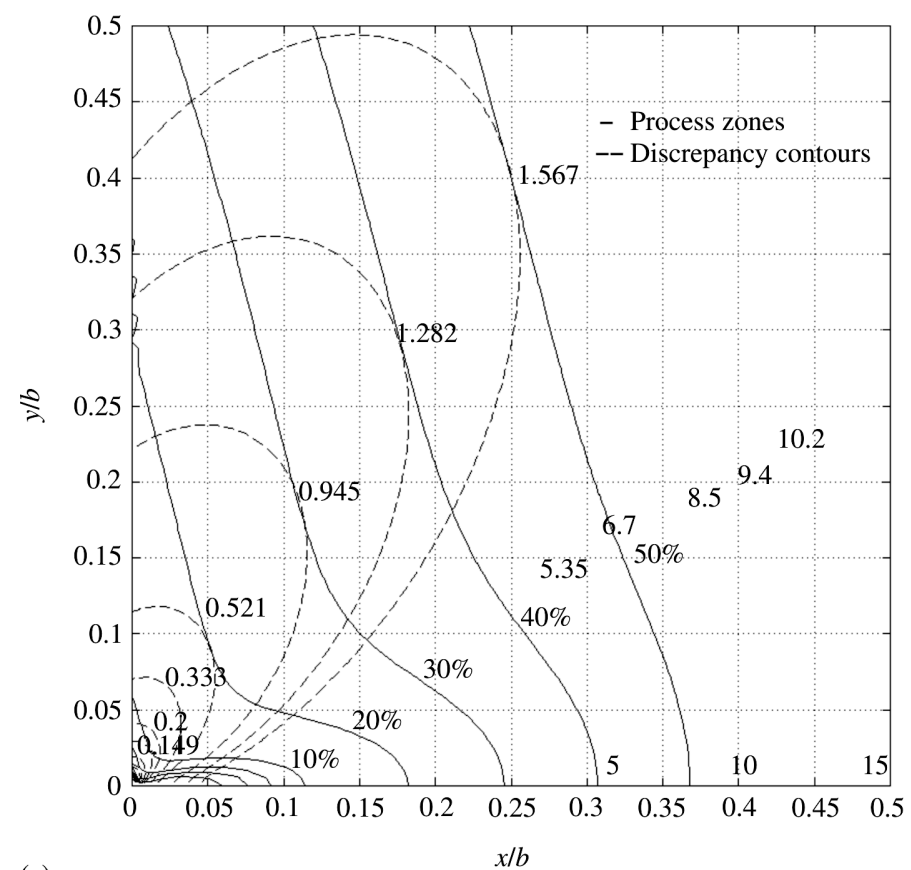

(a)

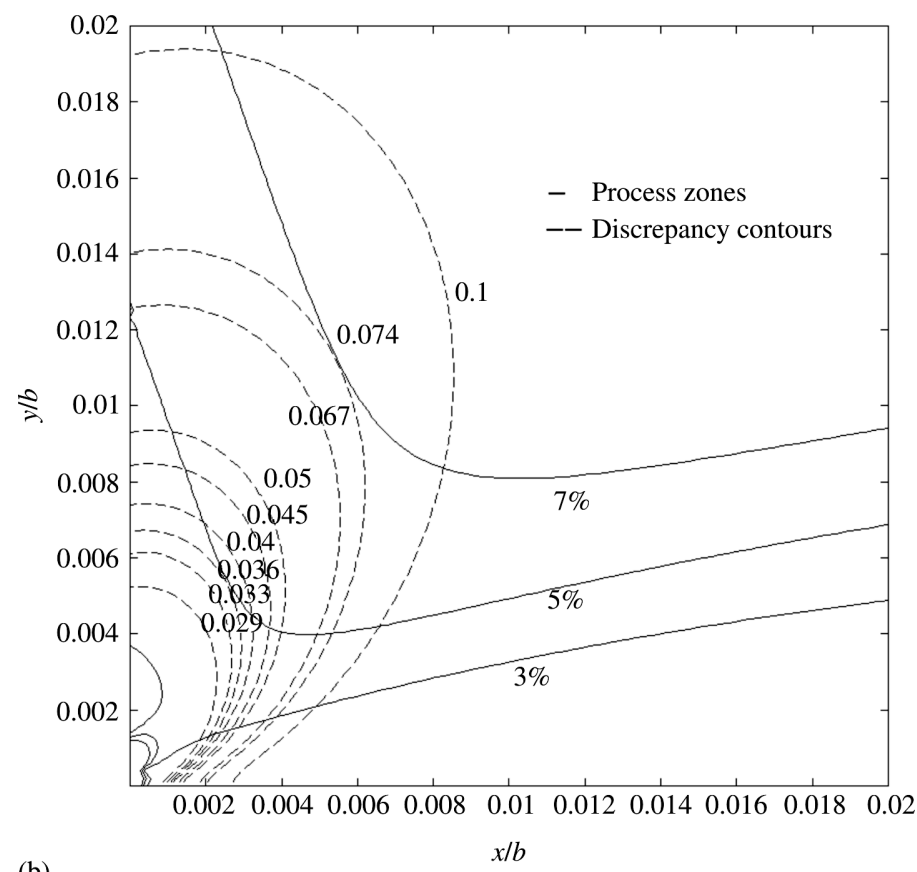

Fig. 3 Discrepancy between solutions A and B together with process zone front locations, for solution A, for a given remote load $\left(\sigma_{0} / k\right)$ starting from the crack tip: (a) global view, (b) close-up

(for a general crack) for mismatch by up to 40 per cent. The point is emphasized that the results are independent of the geometry in which the crack lies - the geometry enters the solution only in the way in which it determines the stress intensity factor. The maximum tolerable root radius, or the maximum amount of blunting that may be accepted, has therefore been determined. 

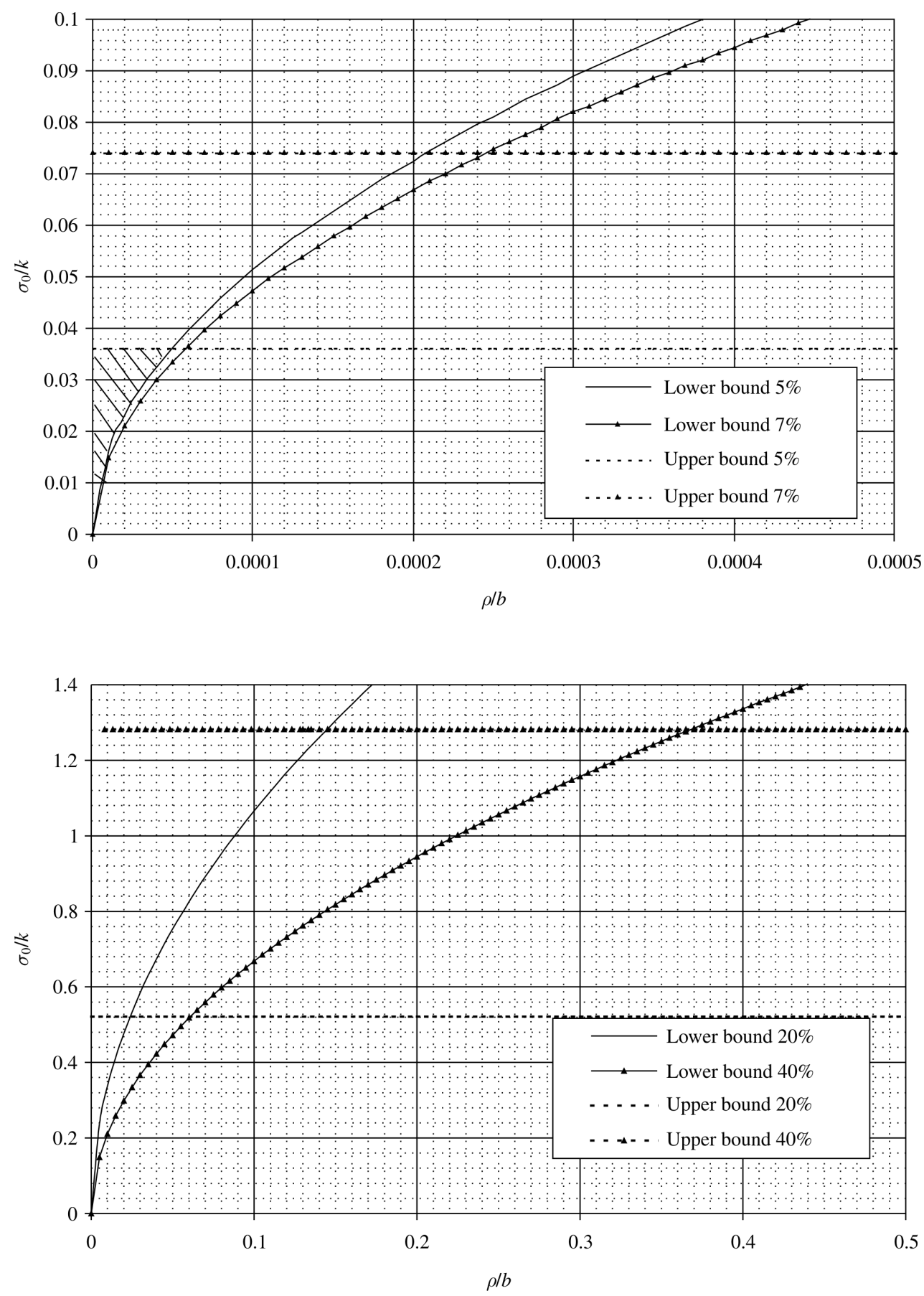

Fig. 4 Range of the permissible normalized load $\left(\sigma_{0} / k\right)$, as a function of $\rho / b$, to achieve characterization of the process zone by the singular solution $\mathrm{B}$ for the case of an edge crack under uniform remote tension, to within a given discrepancy: (a) 5 and 7 per cent; (b) 20 and 40 per cent 


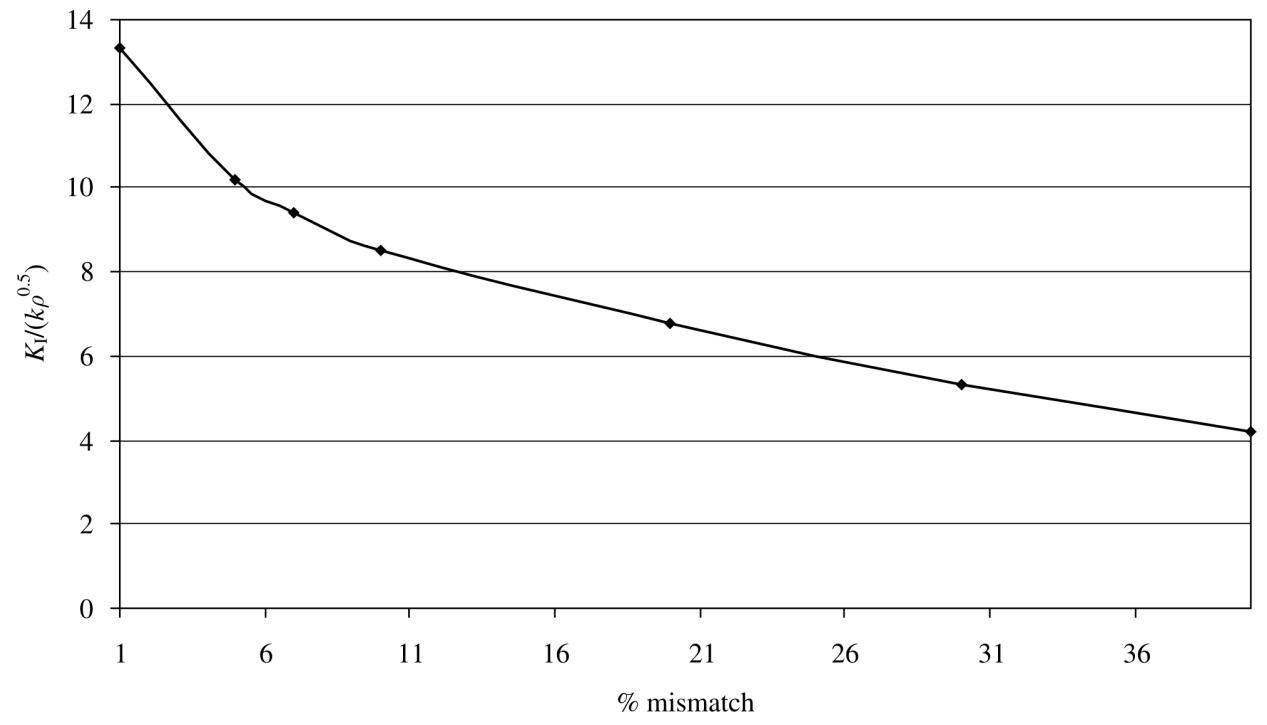

Fig. 5 Lower bound load in the characterization of the process zone

\section{REFERENCES}

1 Kanninen, M. F. and Popelar, C. H. Advanced Fracture Mechanics, 1985 (Oxford University Press, New York).

2 Paris, P. C., Gomez, M. P. and Anderson, W. P. A rational analytic theory of fatigue. Trend in Engng, 1961, 13, 9-14.

3 Irwin, G. R. Analysis of stresses and strains near the end of a crack traversing a plate. J. Appl. Mechanics, 1957, 24, 361-364.

4 Wang, Y. and Pan, J. A plastic fracture mechanics model for characterization of multiaxial low-cycle fatigue. Int. J. Fatigue, 1998, 20(10), 775-784.

5 Evans, W. T. and Luxmore, A. R. Limitations of the Westergaard equations for experimental determination of stress intensity factors. J. Strain Analysis, 1976, 11(3), 177185.

6 Williams, M. L. Stress singularities resulting from various boundary conditions in angular corners of plates in extension. J. Appl. Mechanics, 1952, 24, 109-114.
7 Westergaard, H. M. Bearing pressures and cracks. J. Appl. Mechanics, 1937, 6, A49-A53.

8 Filippi, S., Lazzarin, P. and Tovo, R. Development of some explicit formulas useful to describe elastic stress fields ahead of notches in plates. Int. J. Solids and Structs, 2002, 39, 4543-4565.

9 Nowell, D. and Hills, D. A. Open cracks at or near free edges. J. Strain Analysis, 1987, 22, 177-185.

10 Hills, D. A. and Nowell, D. Mechanics of Fretting Fatigue, 1994 (Kluwer, Dordrecht, The Netherlands).

11 Dundurs, J. and Sendeckyj, G. P. Behaviour of an edge dislocation near a bimetallic interface. J. Appl. Phys., 1965, 36, 3353-3354.

12 Erdogan, F., Gupta, G. D. and Cook, T. S. Numerical solution of singular integral equations. In Methods of Analysis and Solutions of Crack Problems (Ed. G. C. Sih), 1973, pp. 368-425 (Noordhoff, Leyden, The Netherlands).

13 Kaya, A. C. and Erdogan, F. On the solution of integral equations with strongly singular kernels. Q. Appl. Math., 1987, 45, 105-122. 